\title{
Varicella Zoster encephalitis versus Acyclovir toxicity: Diagnostic dilemma and Treatment challenge in an ESRD patient
}

\author{
Tania Mahbub ${ }^{1 *}$, Elena Haque 2 , Salina Akter², Mohammad Omar Faruq ${ }^{3}$ \\ Background: Varicella Zoster (VZ) is of particular concern in end-stage renal disease (ESRD) patients due to their \\ older age (48\% are $>65$ years old) and relatively immunosuppressed state ${ }^{1,2}$. Although older age is the most important \\ risk factor for development of zoster, an immunosuppressed state in the setting of zoster has been shown to have a \\ significantly higher rate of complications. Previous studies have shown that ESRD patients are at increased risk of \\ developing zoster compared with the general population ${ }^{3}$. In this regard, the incidence in the general population is \\ estimated to be 3.9 per 1000 person-years ${ }^{4}$, whereas the incidence in ESRD patients is estimated to be nearly 20-fold \\ higher, at 73.3 per 1000 person-years ${ }^{3}$. Diagnosis and treatment of HZ encephalitis is challenging as sometimes it may \\ resemble as acyclovir toxicity. Early diagnosis is crucial as the treatment of Acyclovir toxicity and VZ encephalitis is \\ totally opposite. Moreover, delay in treatment VZ encephalitis may result increased morbidity and mortality.
}

Here we report a patient who presented with acute confusional state following primary varicella infection.

Case presentation: A 73 years old lady with background history of Diabetes, Hypertension who was on maintenance hemodialysis 3 times per week for 2 years presented to her Nephrologist with confluent maculo papular rash involving right upper hemi thorax which was moderately painful and itchy (Fig. 1) Assuming Varicella Zoster infection oral acyclovir was started empirically after adjustment of renal dose and she was referred to dermatology for further evaluation. However, after increasing the dose of acyclovir to $500 \mathrm{mg}$ thrice daily instead of $200 \mathrm{mg}$ twice daily, as directed by dermatologist, she presented to the emergency with agitation, confusion, hallucination and difficulty in speech after one day. She had no fever, seizure, photophobia and weakness of any part of body. Travelling and contact history was negative and had no previous history of CNS infection. She had no history of prior vaccination with VZ vaccine. She had a temperature of 98.4 degree F, blood pressure of 140/90 $\mathrm{mm} \mathrm{Hg}$, pulse rate of 80 per minute and SPO2 of $98 \%$ at room air. Apart from slurred speech and bilateral equivocal planter response there was no neurological deficit or signs of meningeal irritation. Other general and systemic examination were unremarkable.

Considering VZ encephalitis she was placed on IV acyclovir and the dose adjusted for ESRD hemodialysis patient and she was placed on IV dexamethasone. Meanwhile, routine and specific investigations including CSF study and MRI of brain

1. Consultant, Dept. of Nephrology, United Hospital Ltd, Dhaka 1212, Bangladesh

2. Specialist, Dept. of Nephrology, United Hospital Ltd, Dhaka 1212, Bangladesh.

3. Professor of CCM, Chief Consultant, General ICU and Emergency dept. United Hospital Ltd, Dhaka 1212, Bangladesh.

*Corresponding Author:

Dr. Tania Mahbub

E mail : taniaimran1@yahoo.com were done. Acyclovir neurotoxicity was suspected considering temporal association between symptoms and acyclovir use. Thereby, frequency of hemodialysis was increased. However, Patient condition was deteriorating. When she became deeply unconscious she was transferred to ICU. Later neurological signs developed in the form of bilateral extensor planter response, sluggish pupillary response and partial neck rigidity.

1st sample of CSF showed high protein content and lymphocyte count and it was negative for all neurotropic bacteria and Tuberculosis. Varicella Zoster Virus (VZV) IgM was positive in CSF.

MRI of brain showed degenerative cortical atrophy with micro vascular ischemic changes in sub-cortical white mater of both frontal lobes, corona radiata, periventricular areas and left basal ganglia (Fig 2). Consultations were taken from specialist of Neuromedicine, Critical Care Medicine and Infectious Disease. Decision was taken to continue acyclovir along with dexamethasone and empirical IV Meropenem in renal adjusted dose and hemodialysis was to be continued as per schedule.

Patient improved clinically and eventually became oriented and responsive to verbal command. Repeat CSF showed decreasing protein content and lymphocyte count and follow up MRI was unchanged. VZV IgG was positive in CSF in high titer.

Throughout the period of hospital stay, hemodialysis was continued and she completed IV acyclovir for total three weeks, followed by oral valacyclovir for one week. I/V dexamethasone was switched to oral prednisolone and tapered accordingly. Her functional and neurological status improved back to premorbid level on discharge.

\section{Discussion:}

VZ encephalitis presents with fever, neck rigidity and other focal neurological deficit with or without rash. Whereas, 
acyclovir neurotoxicity presents commonly with visual hallucination and speech disorder.

In our patient, acute confusional state in the back ground of ESRD and having confluent maculopapular rash in dermatomal pattern was more indicative of VZ encephalitis as a disease progression with complication though she did not have fever and focal neurological sign at presentation .Instead, she presented with overlapping symptoms of acute acyclovir neurotoxicity in the form of hallucination and difficulty in speech after an increment in acyclovir dose. Thereby, keeping encephalitis in mind we targeted to treat acyclovir toxicity first by reducing the dose of acyclovir and frequent hemodialysis besides doing CSF study and MRI of brain to exclude viral encephalitis.

Low LL et al reported a case ${ }^{5}$ of 58 year old patient with background of hypertension and ESRD on haemodialysis three times per week who developed vesiculo-papular pruritic lesions mainly over the trunk. He was treated with oral Valacyclovir $500 \mathrm{mg}$ daily. Later patient developed vivid visual hallucinations, became restless, agitated and disorientated to time, place and person. Later he was diagnosed as a case of varicella zoster infection complicated by Varicella encephalitis. Valacyclovir was switched to intravenous acyclovir. A lumbar puncture showed increased protein and lymphocytes. Varicella Zoster Virus (VZV) IgM was positive. MRI of brain showed no evidence of acute infarct, intracranial bleed, space-occupying lesion or hydrocephalus. The frequency of dialysis was increased with continuation of the renal adjusted dose of acyclovir. Throughout the admission, his hemodialysis continued and he completed 2 weeks course of IV acyclovir. His functional status improved back to the pre-morbid levels on discharge.

Abed YWA described another case ${ }^{6}$ of an elderly gentleman with end stage renal disease on haemo dialysis who presented with ophthalmic zoster infection and was discharged on oral acyclovir. He presented again a few days later with confusion and expressive dysphasia. Differential diagnosis was mainly between varicella-zoster associated encephalitis versus acyclovir toxicity. Cerebrospinal fluid analysis confirmed the diagnosis of VZ associated encephalitis and the patient was treated with intravenous acyclovir and steroid with full recovery back to pre-admission neurological status.

Thind et al described ${ }^{7}$ a case of acyclovir neurotoxicity presenting with atypical cerebrospinal fluid findings. An 82-year-old man with a history of end-stage renal disease presented with progressively worsening confusion and somnolence for 4-5 days. The patient was diagnosed with herpes zoster and was placed on a course of valacyclovir $1 \mathrm{~g}$ three times a day (dose not adjusted for renal impairment). MRI of the brain was done and was unremarkable. Although Acyclovir-induced encephalopathy was high on differential, but his CSF findings were consistent with for viral encephalitis. Patient showed immense improvement and eventually recovered completely after dialysis. CSF PCR tests for both herpes simplex virus and varicella zoster virus were found negative.
Daily hemodialysis for extended period may shorten the neurotoxicity period and can help with faster return to normal mentation. A high index of suspicion is warranted to diagnose acyclovir-induced neurotoxicity as sometimes it's difficult to differentiate between VZ encephalitis and acyclovir neurotoxicity especially in patients with renal impairment. In our case we faced the same challenge as there was overlapping clinical and biochemical features. However, patient responded to antiviral and positive CSF VZ IgM and IgG confirmed the diagnosis.

Conclusion and recommendation: Patients treated with long-term hemodialysis are at an increased risk of $\mathrm{VZ}$ infection and fatal complications compared to the general population. Acyclovir is very effective in $\mathrm{VZ}$ infection but it is excreted by the kidneys so dose adjustment is always needed. Neurological symptoms of acyclovir toxicity can mimic VZV associated encephalitis and should always be considered in the differential diagnosis. Due to increasing burden of chronic kidney disease, there should be a consensus guideline on varicella vaccination for renal patients as they suffer from serious consequences of $\mathrm{VZ}$ infection compared to general population.

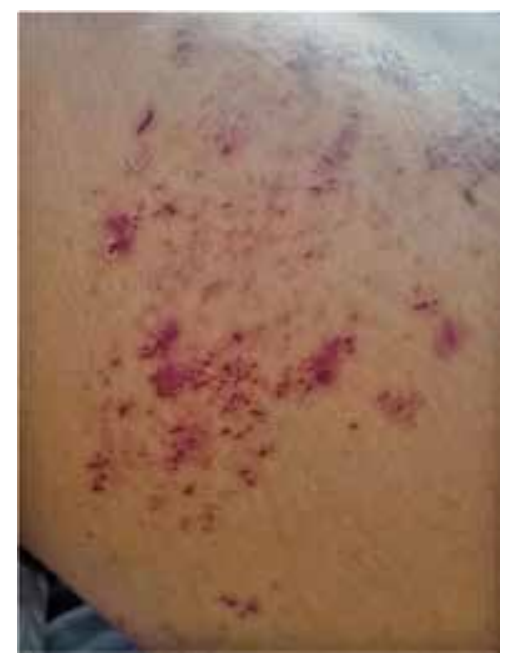

Fig 1

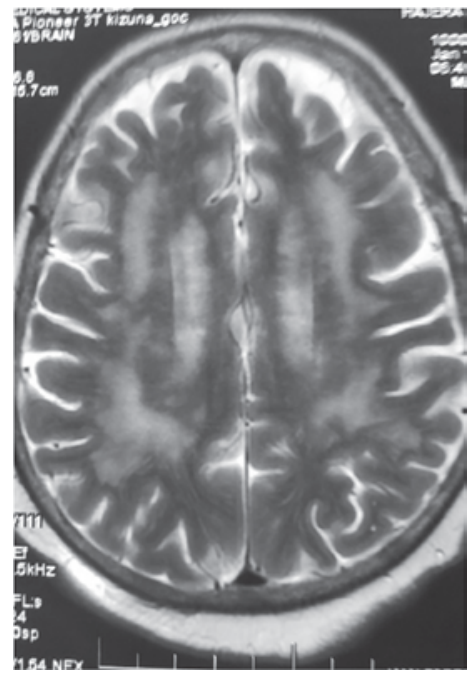

Fig 2 


\section{References:}

1. Meijers RW, Betjes MG, Baan CC et al. T-cell ageing in end stage renal disease patients: assessment and clinical relevance. World J Nephrol 2014; 3: 268-276

2. Betjes MG, Meijers RW, Litjens NH. Loss of renal function causes premature aging of the immune system. Blood Purif 2013; 36: $173-178$

3. Kuo CC, Lee CT, Lee IM et al. Risk of herpes zoster in patients treated with long-term hemodialysis: a matched cohort study. Am J Kidney Dis 2012; 59: 428-433.
4. Robinson ES, Payne AS, Pappas-Taffer L et al. The incidence of herpes zoster in cutaneous lupus erythematosus (CLE), dermatomyositis (DM), pemphigus vulgaris (PV), and bullous pemphigoid (BP). J Am Acad Dermatol 2016; 75: 42-48

5. Leng LL, Vasanwala FF, Suhail SM. "Varicella encephalitis and pneumonia in a patient with end stage renal failure." Asia Pacific Family Medicine, vol. 13, no. 1, 2014.

6. Abed YWA. Varicella-zoster virus associated encephalitis in a patient undergoing haemodialysis. Qatar Medical Journal, 01 Jan 2015, 2015(2):19

7. Thind GS, Roach R. A case of acyclovir neurotoxicity presenting with atypical cerebrospinal fluid findings, BMJ Journal, 2017 